\title{
Prognostic value of coronary artery calcium score in symptomatic individuals: A meta-analysis of 34,000 subjects is
}

\author{
Mallory S. Lo-Kioeng-Shioe a, Dorine Rijlaarsdam-Hermsen ${ }^{\mathrm{a}, \mathrm{b}}$, Ron T. van Domburg a , Martin Hadamitzky ${ }^{\mathrm{c}}$, \\ João A.C. Lima ${ }^{\mathrm{d}}$, Sanne E. Hoeks ${ }^{\mathrm{a}}$, Jaap W. Deckers ${ }^{\mathrm{a}, *}$ \\ a Department of Cardiology, Erasmus MC, University Medical Center, Rotterdam, the Netherlands \\ ${ }^{b}$ Medical Center Haaglanden Bronovo, The Hague, the Netherlands \\ c Institut für Radiologie und Nuklearmedizin, Hospital at the Technische Universität München, Munich, Germany \\ d Department of Cardiology, Johns Hopkins Hospital and School of Medicine, Baltimore, MD, USA
}

\section{A R T I C L E I N F O}

\section{Article history:}

Received 2 December 2018

Received in revised form 18 April 2019

Accepted 1 June 2019

Available online $\mathrm{xxxx}$

\section{Keywords:}

Coronary artery disease

Coronary artery calcium

Prognosis

Computed tomography

Cardiovascular events

\begin{abstract}
A B S T R A C T
Background: Coronary artery calcium (CAC) scanning has evolved into an important subclinical prediction method for cardiovascular diseases in asymptomatic subjects. However, the prognostic implication of CAC scanning in symptomatic individuals is less clear.

Objectives: To assess the prognostic utility of CAC in predicting risk of major adverse cardiac events (MACE) in stable patients with suspected CAD.

Methods: We did a systematic electronic literature search for studies presenting original data in CAC score, and reporting cardiovascular events in stable, symptomatic patients as primary outcome. Primary outcome of the meta-analysis was the occurrence of MACE, a composite of late coronary revascularization, hospitalization for unstable angina or heart failure, nonfatal myocardial infarction, and cardiac death or all-cause mortality. Using random effects models, we pooled relative risk ratios of CAC for MACE, and adjusted hazard ratios (HR) of the associations between different CAC strata (CAC 0-100,100-400, and $\geq 400$, versus CAC $=0$ ) and incident MACE. Results: We included 19 observational studies $(n=34,041)$. In total, 1601 events were analyzed, of which 158 in patients with $C A C=0$. The pooled relative risk ratio was $5.71(95 \%-\mathrm{Cl}: 3.98 ; 8.19)$ for subjects with $\mathrm{CAC}>0$. The pooled estimate of adjusted HRs demonstrated increasing, positive associations, with the strongest association for CAC > 400 (HR: 4.88; 95\%-CI: 2.44;9.27).

Conclusions: This meta-analysis demonstrated that increased levels of CAC are strongly and independently associated with increased risk for MACE in stable, symptomatic patients with suspected CAD, showing increasing risk with greater CAC scores. Application of CAC scanning as a prediction method could be useful for a considerable number of such patients.
\end{abstract}

(C) 2019 Published by Elsevier B.V.

\section{Introduction}

During the past two decades, coronary artery calcium (CAC) scanning has evolved into an important prediction method for cardiovascular diseases (CVD) [1,2]. In combination with traditional risk factors, CAC can be used to calculate an accurate estimate of the 10-year coronary heart disease risk in men and women without symptoms [3]. For these reasons, the 2013 ACCF/AHA Risk Assessment Guidelines has given CAC scanning Class IIa and IIb recommendations for the

\footnotetext{
it All authors take responsibility for all aspects of the reliability and freedom from bias of the data presented and their discussed interpretation.

* Corresponding author at: Thoraxcenter, Division of Cardiology, Erasmus MC, P.O. Box 2040, 3000 CA Rotterdam, the Netherlands.

E-mail address: j.deckers@erasmusmc.nl (J.W. Deckers).
}

evaluation of CVD risk in intermediate and low-to-intermediate asymptomatic subjects, respectively [4,5].

While CAC testing is mainly done in asymptomatic individuals, several studies have evaluated the prognostic value of CAC in symptomatic patients. However, the implication of CAC testing in such subjects is less clear, as a result of heterogeneity of the patient groups studied. Some studies included (solely) patients referred for diagnostic evaluation in suspected CAD, while others investigated patients self-referred to an emergency department for acute symptoms, or a combination of both. Of note, the majority of meta-analyses published to date have included either asymptomatic patients or patients in different clinical settings, and thus provide data that cannot be extrapolated easily to stable symptomatic patients [6-9]. Hence, the aim of this paper was to provide a comprehensive overview of the existing literature on the prognostic value of CAC in a large group of patients with stable symptoms. 
We conducted a systematic review and study-based meta-analysis on the prognostic utility of CAC in predicting risk of major adverse cardiac events (MACE) in stable patients presenting with suspected coronary artery disease (CAD). This paper thus describes risk ratios for a composite outcome of MACE for patients with positive CAC scores relative to subjects without CAC.

\section{Methods}

2.1. Data sources and search strategy

We conducted a meta-analysis using the Meta-analysis of Observational Studies in Epidemiology (MOOSE) guidelines [10]. Together with an experienced librarian (W.B.), we searched EMBASE, Ovid MEDLINE, Web of Science, Cochrane CENTRAL and Google Scholar until September 2017. A complete overview of the search terms can be found in the online data supplement. No restrictions on language or date were applied. In case of potentially eligible articles with a different alphabet assistance was sought from native speakers or digital translation resources. Additionally, we screened the bibliographies of key articles for potentially eligible records.

\subsection{Study selection}

We included studies that presented original data on CAC score, and that reported cardiovascular events as primary outcome. All studies had to provide either absolute numbers of events in patient groups according to their CAC status or cox proportional hazard ratios (HRs) for the prediction of events. In addition, the patient population should comprise stable symptomatic patients with a suspicion of CAD in at least $50 \%$ of the population studied. We excluded studies in which $\geq 5 \%$ of the population had a known history of CAD at baseline. In addition, we excluded studies including symptomatic patients examined in an emergency care setting.

\subsection{Data extraction and quality assessment}

Two independent investigators (M.S.L. and J.W.D.) examined the titles and abstracts of the reports retrieved through the database search. Reports that appeared to meet the inclusion criteria were assessed in detail by reviewing the full-texts. We contacted authors by email if they did not report absolute numbers of events per CAC status. We extracted data on study design, clinical characteristics of participants, follow-up duration, sample size, number of events according to patients' CAC status, pre-test likelihood of CAD, CT scanner, and timing of patient enrollment. In studies reporting multiple cardiac events per patient, only one event was counted as MACE.

The quality of the included studies was assessed using Hayden's quality appraisal for prognostic studies [11]. Domains of potential biases that were relevant for our metaanalysis included [1] study participation, [2] prognostic factor measurement, and [3] outcome measurement. We examined these domains using the corresponding guidelines.

\subsection{Endpoints}

The outcome of our meta-analysis was the occurrence of MACE, defined as the composite incidence of any of the following: late cardiac revascularization (coronary artery bypass graft or percutaneous coronary intervention), hospitalization for unstable angina pectoris or heart failure, nonfatal myocardial infarction, and cardiac death or all-cause mortality. Furthermore, we investigated the relationship between the presence of CAC and the composite of nonfatal myocardial infarction and all-cause mortality.

\subsection{Statistical analysis}

In studies that reported the events per CAC stratum, we calculated the MantelHaenszel individual and summary relative risk ratios and $95 \%$ confidence intervals, comparing the relative risks for different CAC score reference groups ( $C A C=0, C A C<100$, and $C A C<400$ ). We used the event data in patients with $C A C>0$ versus $C A C=0$ to calculate relative risk ratios of CAC presence.

For each study, the annual event rate per 100 patients with was $C A C=0$ was approximated by dividing the proportion of $C A C=0$ patients with an event by the follow-up duration in months and multiplying this by 12 .

Furthermore, we pooled adjusted HRs of the associations between different CAC strata (CAC $0-100$ vs $C A C=0$, CAC $100-400$ vs $C A C=0$, and $C A C \geq 400$ vs $C A C=0$ ) and incident MACE.

Meta-analyses were done using both random and fixed effects modelling. Results are presented in Forest plot illustrations and summarized in tables. We evaluated presence of heterogeneity by calculating Cochran's Q statistic and the tau-squared, and quantified the heterogeneity using the $\mathrm{I}^{2}$ index. We visually examined funnel plot symmetry to assess publication bias. In the absence of publication bias a funnel plot should approximately resemble a symmetrical inverted funnel. In the presence of pronounced asymmetry, publication bias may be present. All statistical analyses were done using Rstudio software (Version 1.0.153, Meta, Metafor, Metagen).

\section{Results}

\subsection{Study population}

We identified 5281 reports through electronic database searches and 4 additional reports through manual searching of bibliographies of key articles (Fig. S1). We excluded 2928 reports on the basis of title and 2256 on the basis of abstract. There were no eligible manuscripts with a different alphabet. This yielded 101 potentially relevant reports of which we retrieved full-texts. After detailed assessment of these manuscripts, 17 of 101 reports were found to be eligible for our systematic review. Email correspondence with authors of publications that did not report absolute numbers of events per CAC status led to inclusion of two additional reports [12,13]. Our subsequent total of 19 reports provided information on 34,041 participants. Four of the 19 reports were multicenter studies. All studies were observational and were published between 1996 and 2016, with a follow-up duration that varied from 17 to 82 months. The proportion of women included ranged from $19 \%$ to $68 \%$. Between $30 \%$ and $74 \%$ of participants presented with hypertension at baseline, between $7 \%$ and $55 \%$ with diabetes. In 17 out of 19 reports patients with a known history of CAD were excluded [12,14-28]. Among the 12 studies reporting on family history of CAD, its prevalence ranged from $27 \%$ to $66 \%$. The number of participants with a CAC score of zero varied between $11 \%$ and $63 \%$. Among the four studies reporting lipid-lowering therapy, its prevalence ranged from $15.8 \%$ to $63 \%$ $[16,19,23,25]$. Eight out of 19 studies specified type of chest pain (i.e. typical angina, atypical angina or nonanginal chest pain) $[12,14,16,18,20,21,23,27]$. The annual event rate per 100 patients with $C A C=0$ varied between 0 and 3.64 (Table 1 ). In addition to CAC presence, 11 studies assessed the extent of coronary lumen stenosis by coronary computed tomography angiography (CCTA). The proportion of participants with obstructive CAD (defined as $>50 \%$ lumen stenosis) varied between $14 \%$ and $72 \%$ (Table 2 ).

\subsection{Cardiovascular events}

In total, 1601 (4.7\%) cardiovascular events were analyzed, 158 (1.18\%) of these events occurred in patients with a CAC score of zero. Four studies reported multiple events per study participant, for these studies we used the number of patients in whom an event occurred $[14,18,25,26]$. Eighteen $[12-21,23-30]$ of 19 studies assessed the occurrence of cardiovascular events stratified per CAC status (CAC $>0$ vs CAC $=0$ ). Only one study reported HRs per CAC stratum [22]. The pooled relative risk ratio for MACE in patients with any detectable CAC was 5.71 (95\% confidence interval [CI]: 3.98 to $8.19, \mathrm{I}^{2}: 65 \%$ ) using random effects models (Table 3, Fig. 1a). Thirteen studies presented data on the occurrence of either all-cause mortality or nonfatal MI, or a combination per CAC status in addition to overall events. The pooled analysis for incidence of all-cause mortality or nonfatal myocardial infarction gave a relative risk ratio of 3.64 (95\% CI: 2.68 to $4.96, \mathrm{I}^{2}: 16 \%$ ) (Fig. 1b). The metaanalyses comparing different CAC score reference groups are shown in Table 3. Using a reference group of $C A C=0$, both a threshold of $C A C$ $\geq 100$ and $\geq 400$ yielded an increased cumulative relative risk for MACE.

Pooled estimates of HRs adjusted for clinical risk factors demonstrated increasing and positive associations for increasing CAC strata and risk of future events, the strongest association being found in patients with CAC > 400 (HR 4.88, 95\% CI: 2.44 to 9.76, $\mathrm{I}^{2}$ : 69.8\%) (Online data supplement: Table S1).

A sensitivity analysis was done for the twelve studies [12,14-19,24,26-29] in which $100 \%$ of the patients were reported to be symptomatic. Among these 20,421 patients, the pooled relative risk ratio for incident MACE was 5.85 (95\% CI: 3.69 to 9.27, $\mathrm{I}^{2}$ : $54 \%)$. 
Table 1

Characteristics of included studies.

\begin{tabular}{|c|c|c|c|c|c|c|c|c|c|c|}
\hline Author, year & Design & $\begin{array}{l}\text { Total } \\
\text { population }\end{array}$ & $\begin{array}{l}\text { Follow-up, } \\
\text { months }\end{array}$ & $\begin{array}{l}\text { Loss of } \\
\text { follow-up } \\
(\%)\end{array}$ & Definition of events & $\begin{array}{l}\text { Events } \\
\mathrm{CAC}= \\
0\end{array}$ & All & $\begin{array}{l}\text { Events, } \\
\%\end{array}$ & $\begin{array}{l}\text { Annual } \\
\text { event } \\
\text { rate, } \\
\text { per } 100 \\
\text { CAC = } \\
0 \\
\text { patients }\end{array}$ & $\begin{array}{l}\text { Scanner } \\
\text { type/slice } \\
\text { thickness }\end{array}$ \\
\hline $\begin{array}{l}\text { Al-Mallah, } \\
2014[14]\end{array}$ & Multicenter & 8627 & $\begin{array}{l}25 \\
\text { (median) }\end{array}$ & $66(0.8 \%)$ & Death, nonfatal MI & 31 & 150 & $2 \%$ & 0.31 & $\begin{array}{l}\text { MDCT } \\
(\geq 64)\end{array}$ \\
\hline $\begin{array}{l}\text { Becker, } 2005 \\
\text { [15] }\end{array}$ & Single-center & 924 & 35 (mean) & NR & Cardiac death, MI & 0 & 78 & $8 \%$ & 0 & $\begin{array}{l}\mathrm{MDCT} / 3 \\
\mathrm{~mm}\end{array}$ \\
\hline Bom, 2016 [16] & Single-center & 1551 & $\begin{array}{l}21 \\
\text { (median) }\end{array}$ & $0.6 \%$ & Death, MI, late revascularization & 2 & 23 & $1 \%$ & 0.15 & $\begin{array}{l}\text { MDCT }(64, \\
128)\end{array}$ \\
\hline $\begin{array}{l}\text { Chaikriangkrai, } \\
2015 \text { [17] }\end{array}$ & Single-center & 805 & 28 (mean) & $151(16 \%)$ & Cardiac death, nonfatal MI, late revascularization & 0 & 25 & $3 \%$ & 0 & $\begin{array}{l}\text { MDCT } \\
(64) / 2.5 \\
\mathrm{~mm}\end{array}$ \\
\hline $\begin{array}{l}\text { Dedic, } 2011 \\
\quad[18]\end{array}$ & Single-center & 424 & 31 (mean) & $47(10 \%)$ & $\begin{array}{l}\text { Cardiac death, nonfatal MI, unstable angina requiring } \\
\text { hospitalization, late revascularization }\end{array}$ & 2 & 30 & $10 \%$ & 0.51 & $\begin{array}{l}\text { MDCT } \\
{[32] / 3 \mathrm{~mm}}\end{array}$ \\
\hline $\begin{array}{l}\text { Detrano, } 1996 \\
\text { [29] }\end{array}$ & Multicenter & 491 & 30 (mean) & $69(14 \%)$ & Cardiac death, nonfatal MI & 1 & 21 & $4 \%$ & 0.41 & EBCT \\
\hline $\begin{array}{l}\text { Engbers, } 2016 \\
\text { [19] }\end{array}$ & Single-center & 4897 & $\begin{array}{l}31 \\
\text { (median) }\end{array}$ & $8(0.2 \%)$ & Death, nonfatal MI, late revascularization & 21 & 278 & $6 \%$ & 0.62 & $\begin{array}{l}\mathrm{MDCT} / 2.5 \\
\mathrm{~mm}\end{array}$ \\
\hline $\begin{array}{l}\text { Hadamitzky, } \\
2011[13]\end{array}$ & Single-center & 2223 & $\begin{array}{l}28 \\
\text { (median) }\end{array}$ & $96(4.1 \%)$ & $\begin{array}{l}\text { Cardiac death, nonfatal MI, unstable angina requiring } \\
\text { hospitalization, late revascularization }\end{array}$ & 2 & 47 & $2 \%$ & 0.10 & $\begin{array}{l}\text { MDCT }(16, \\
64)\end{array}$ \\
\hline Hou, $2012[20]$ & Single-center & 4425 & $\begin{array}{l}35 \\
\text { (median) }\end{array}$ & $\begin{array}{l}582 \\
(11.6 \%)\end{array}$ & Cardiac death, MI, late revascularization & 56 & 363 & $8 \%$ & 0.69 & MDCT (64) \\
\hline $\begin{array}{l}\text { Hulten, } 2014 \\
\quad[12]\end{array}$ & Multicenter & 1145 & $\begin{array}{l}28 \\
\text { (median) }\end{array}$ & NR & Cardiac death, MI, angina, late revascularization & 4 & 35 & $3 \%$ & 0.36 & $\begin{array}{l}\text { MDCT } \\
(\geq 64)\end{array}$ \\
\hline $\begin{array}{l}\text { Keelan, } 2001 \\
\quad[30]\end{array}$ & Single-center & 288 & 82 (mean) & $29(9.1 \%)$ & Cardiac death, nonfatal MI & 1 & 22 & $8 \%$ & 0.46 & EBCT \\
\hline $\begin{array}{l}\text { Kunita, } 2014 \\
\quad[21]\end{array}$ & Single-center & 722 & 44 (mean) & NR & Cardiac death, nonfatal MI, late revascularization & 3 & 37 & $5 \%$ & 0.31 & $\begin{array}{l}\text { MDCT }(16, \\
64) / 2.5 \\
\mathrm{~mm}\end{array}$ \\
\hline $\begin{array}{l}\text { Kwon, } 2011 \\
\text { [22] }\end{array}$ & Single-center & 3979 & 27 (mean) & $6(0.1 \%)$ & $\begin{array}{l}\text { Cardiac death, nonfatal myocardial infarction, } \\
\text { unstable angina requiring hospitalization, late } \\
\text { revascularization }\end{array}$ & NR & 105 & $3 \%$ & - & $\begin{array}{l}\text { MDCT } \\
(64) / 3 \mathrm{~mm}\end{array}$ \\
\hline Lee, 2016 [23] & Multicenter & 1077 & $\begin{array}{l}23 \\
\text { (median) }\end{array}$ & $36(3.2 \%)$ & $\begin{array}{l}\text { Cardiac death, nonfatal MI, unstable angina, late } \\
\text { revascularization }\end{array}$ & 6 & 71 & $7 \%$ & 0.93 & $\begin{array}{l}\text { MDCT } \\
(64) / 3 \mathrm{~mm}\end{array}$ \\
\hline Liu, 2013 [24] & Single-center & 98 & 60 (mean) & $2(2 \%)$ & $\begin{array}{l}\text { Cardiac death, unstable angina requiring } \\
\text { revascularization }\end{array}$ & 2 & 56 & $57 \%$ & 2.67 & $\begin{array}{l}\text { MDCT } \\
(64) / 3 \mathrm{~mm}\end{array}$ \\
\hline Naya, 2013 [25] & Single-center & 901 & $\begin{array}{l}18 \\
\text { (median) }\end{array}$ & $71(7.9 \%)$ & $\begin{array}{l}\text { Cardiac death, MI, heart failure requiring } \\
\text { rehospitalization, late revascularization }\end{array}$ & 15 & 57 & $7 \%$ & 2.49 & $\begin{array}{l}\text { MDCT } \\
(64) / 3 \mathrm{~mm}\end{array}$ \\
\hline $\begin{array}{l}\text { Parma, } 2016 \\
\text { [26] }\end{array}$ & Single-center & 588 & 21 (mean) & NR & Cardiac death, nonfatal MI, revascularization & 0 & 108 & $20 \%$ & 0 & $\begin{array}{l}\text { MDCT } \\
(64) / 2 \mathrm{~mm}\end{array}$ \\
\hline $\begin{array}{l}\text { Schenker, } 2008 \\
\text { [27] }\end{array}$ & Single-center & 621 & 17 (mean) & 0 & Death, nonfatal MI & 11 & 55 & $9 \%$ & 3.64 & $\begin{array}{l}\text { MDCT } \\
{[16] / 2.5} \\
\mathrm{~mm}\end{array}$ \\
\hline $\begin{array}{l}\text { Schmermund, } \\
2004[28]\end{array}$ & Single-center & 255 & 38 (mean) & $45(15 \%)$ & Cardiac death, MI, revascularization & 1 & 40 & $16 \%$ & 0.51 & $\begin{array}{l}\mathrm{EBCT} / 3 \\
\mathrm{~mm}\end{array}$ \\
\hline
\end{tabular}

\subsection{Publication bias assessment}

Fig. S2 shows the funnel plot of the studies included in our metaanalysis and might indicate slight asymmetry and subsequent reporting bias with smaller studies showing greater treatment effects.

\subsection{Quality}

We assessed the quality of the included publications on three different domains. In total, we assessed thirteen quality items from the following domains of potential bias: [1] study participation [2], prognostic factor measurement, and [3] outcome measurement. On seven of the thirteen quality items, all 19 reports reached a maximum score. All reports provided an adequate description of the population of interest, and all provided a clear description the prognostic factor, i.e. the CAC score. However, five reports did not fully provide a clear definition of the outcome of interest (Online data supplement: Table S2).

\section{Discussion}

Our extensive systematic literature search and analysis showed that in symptomatic patients with suspected coronary artery disease, presence of CAC significantly predicted MACE as well as the composite outcome of all-cause mortality and nonfatal myocardial infarction. Importantly, these results were not modified by adjustment for clinical risk factors including age, male gender, hypertension, diabetes, dyslipidemia, and current smoking. The absence of CAC was associated with a low risk of future events. In addition to the overall presence of CAC, we compared the risk of MACE at different calcium score thresholds. Compared to a reference group with a CAC score of zero, patients with scores above higher thresholds of CAC (CAC $\geq 100$ and $\geq 400$ ) were at greatest risk for future events. Strata with higher CAC scores were positively and significantly associated with greater risk of incident MACE, also after adjusting for clinical risk factors. All in all, CAC testing proved to be of significant prognostic value for risk stratification in this symptomatic population with suspected CAD. 
Table 2

Patient characteristics.

\begin{tabular}{|c|c|c|c|c|c|c|c|c|c|c|c|c|c|c|c|}
\hline Author, year & $\begin{array}{l}\text { Mean } \\
\text { age }\end{array}$ & $\begin{array}{l}\text { Women, } \\
\%\end{array}$ & $\begin{array}{l}\text { BMI } \\
(\mathrm{kg} / \mathrm{m} 2)\end{array}$ & $\begin{array}{l}\text { HTN, } \\
\%\end{array}$ & $\begin{array}{l}\text { DM, } \\
\%\end{array}$ & DLP, \% & $\begin{array}{l}\text { Cig., } \\
\%\end{array}$ & $\begin{array}{l}\text { CAD } \\
\text { FAM, \% }\end{array}$ & $\begin{array}{l}>50 \% \\
\text { stenosis, \% }\end{array}$ & $\begin{array}{l}\mathrm{CAC}= \\
0\end{array}$ & $\begin{array}{l}\text { CAC } \\
\geq 400\end{array}$ & $\begin{array}{l}\text { Low } \\
\text { P (D } \\
+)\end{array}$ & $\begin{array}{l}\text { Interm. } \\
\mathrm{P}(\mathrm{D}+)\end{array}$ & $\begin{array}{l}\text { High } \\
\text { P (D } \\
+)\end{array}$ & Scoring method \\
\hline Al-Mallah, 2014 [14] & 57 & $50 \%$ & 27.5 & $50 \%$ & $13 \%$ & $56 \%$ & $17 \%{ }^{\mathrm{b}}$ & $30 \%$ & unk & $56 \%$ & $8 \%$ & $49 \%$ & $42 \%$ & $9 \%$ & $\begin{array}{l}\text { Diamond \& } \\
\text { Forrester }\end{array}$ \\
\hline Becker, 2005 [15] & 59 & $52 \%$ & NR & $55 \%$ & $25 \%$ & $\begin{array}{l}44 \% \\
\text { (HLP) }\end{array}$ & $35 \%$ & $66 \%$ & unk & $20 \%$ & NR & NR & & & \\
\hline Bom, 2016 [16] & 58 & $62 \%$ & 26.6 & $30 \%$ & $8 \%$ & $\begin{array}{l}25 \% \\
\text { (HLP) }\end{array}$ & $18 \%$ & $47 \%$ & $14 \%$ & $48 \%$ & $7 \%$ & $34 \%$ & $49 \%$ & $17 \%$ & $\begin{array}{l}\text { Duke Clinical } \\
\text { Score }\end{array}$ \\
\hline $\begin{array}{l}\text { Chaikriang-krai, } \\
2015 \text { [17] }\end{array}$ & 58 & $47 \%$ & NR & $47 \%$ & $14 \%$ & $31 \%$ & $21 \%$ & NR & $15 \%$ & $44 \%$ & $15 \%$ & NR & & & \\
\hline Dedic, 2011 [18] & 56 & $49 \%$ & 26.8 & $51 \%$ & $14 \%$ & $59 \%$ & $28 \%$ & $45 \%$ & $32 \%$ & $36 \%$ & $15 \%$ & NR & & & \\
\hline Detrano, 1996 [29] & 55 & $43 \%$ & NR & NR & NR & NR & NR & NR & $43 \%$ & $20 \%$ & NR & NR & & & \\
\hline Engbers, 2016 [19] & 61 & $57 \%$ & 27.6 & $61 \%$ & $13 \%$ & $\begin{array}{l}43 \% \\
(\mathrm{HCL})\end{array}$ & $16 \%{ }^{\mathrm{b}}$ & $55 \%$ & unk & $27 \%$ & $20 \%$ & $9 \%$ & $91 \%$ & & $\begin{array}{l}\text { Diamond \& } \\
\text { Forrester }\end{array}$ \\
\hline $\begin{array}{l}\text { Hadamitzky, } 2011 \\
\text { [13] }\end{array}$ & $60^{\mathrm{a}}$ & $33 \%$ & 25.7 & $59 \%$ & $8 \%$ & $\begin{array}{l}52 \% \\
\text { (HLP) }\end{array}$ & $35 \%$ & $32 \%$ & $29 \%$ & $37 \%$ & $12 \%$ & $20 \%$ & $74 \%$ & $7 \%$ & Morise \\
\hline Hou, 2012 [20] & 60 & $38 \%$ & 24.1 & $57 \%$ & $15 \%$ & $27 \%$ & $31 \%$ & $35 \%$ & $20 \%$ & $63 \%$ & $8 \%$ & NR & & & \\
\hline Hulten, 2014 [12] & 55 & $37 \%$ & NR & $52 \%$ & $16 \%$ & $\begin{array}{l}55 \% \\
(\mathrm{HLP})\end{array}$ & $15 \%{ }^{\mathrm{b}}$ & $61 \%$ & $25 \%$ & $42 \%$ & NR & & & & Morise, mean: 47 \\
\hline Keelan, 2001 [30] & 56 & $23 \%$ & NR & $45 \%$ & $13 \%$ & NR & $62 \%^{\mathrm{c}}$ & $51 \%$ & NR & $11 \%$ & NR & NR & & & \\
\hline Kunita, 2014 [21] & 65 & $39 \%$ & 23.7 & $58 \%$ & $33 \%$ & $\begin{array}{l}45 \% \\
(\mathrm{HCL})\end{array}$ & $31 \%$ & NR & unk & $36 \%$ & $12 \%$ & NR & & & \\
\hline Kwon, 2011 [22] & 60 & $47 \%$ & 24.6 & $50 \%$ & $16 \%$ & $38 \%$ & $12 \%^{\mathrm{b}}$ & NR & $15 \%$ & $61 \%$ & $5 \%$ & $29 \%$ & $65 \%$ & $6 \%$ & ACC/AHA \\
\hline Lee, 2016 [23] & 62 & $37 \%$ & 25 & $42 \%$ & $55 \%$ & $45 \%$ & $39 \%$ & NR & $46 \%$ & $31 \%$ & $17 \%$ & $32 \%$ & $45 \%$ & $23 \%$ & $\begin{array}{l}\text { Framingham Risk } \\
\text { Score }\end{array}$ \\
\hline Liu, 2013 [24] & 63 & $19 \%$ & NR & $61 \%$ & $22 \%$ & $\begin{array}{l}27 \% \\
(\mathrm{HCL})\end{array}$ & $14 \%$ & NR & $72 \%$ & $15 \%$ & $44 \%$ & NR & & & \\
\hline Naya, 2013 [25] & $61^{\mathrm{a}}$ & $68 \%$ & 30.2 & $74 \%$ & $31 \%$ & $54 \%$ & $11 \%$ & $27 \%$ & unk & $44 \%$ & $15 \%$ & NR & $51 \%$ & NR & $\begin{array}{l}\text { Duke Clinical } \\
\text { Score }\end{array}$ \\
\hline Parma, 2016 [26] & 61 & $64 \%$ & 28.2 & $76 \%$ & $15 \%$ & $\begin{array}{l}57 \% \\
(\mathrm{HCL})\end{array}$ & $21 \%^{\mathrm{c}}$ & $61 \%$ & unk & $40 \%$ & $12 \%$ & NR & & & \\
\hline Schenker, 2008 [27] & 61 & $60 \%$ & 32.4 & $74 \%$ & $28 \%$ & $\begin{array}{l}54 \% \\
\text { (HLP) }\end{array}$ & $14 \%$ & $37 \%$ & unk & $34 \%$ & $25 \%$ & & $100 \%$ & & NR (mean: 59.4\%) \\
\hline $\begin{array}{l}\text { Schmermund, } 2004 \\
\text { [28] }\end{array}$ & 58 & $29 \%$ & NR & $41 \%$ & $7 \%$ & $\begin{array}{l}57 \% \\
(\mathrm{HCL})\end{array}$ & $19 \%^{\mathrm{b}}$ & NR & $37 \%$ & $24 \%$ & NR & NR & & & \\
\hline
\end{tabular}

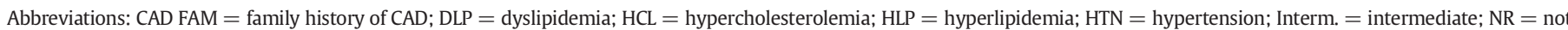
reported; $\mathrm{P}(\mathrm{D}+)=$ pre-test likelihood; unk = unknown.

a Median age.

b Current smoking.

c Any history of smoking.

Ours is not the first meta-analysis of CAC scanning in symptomatic subjects. In 2009, Sarwar et al. described the prognostic value of CAC absence in symptomatic patients [31]. Based on their pooled analysis of 7 studies including almost 4000 patients, the cumulative relative risk ratio for patients with a CAC score of zero as compared with patients with positive CAC scores was found to be 0.09 , concordant with our results. Nonetheless, our meta-analysis differs from this previous analysis in several aspects. First, we were able to include 13 new studies that additionally contributed over 31,000 patients $[12-14,16-23,25,26]$. Second, we excluded studies on patients presenting with acute symptoms and, third, uphold a higher threshold for CAD suspicion. These actions resulted in a better defined and more homogenous study population. Fourth, we analyzed both the prognostic value of CAC per CAC status (CAC $>0$ versus $C A C=0$ ), as well as per different thresholds of CAC and CAC strata. Fifth and last, while a large number of events in the published literature

Table 3

Meta-analysis comparing risk for MACE at different thresholds of CAC.

\begin{tabular}{|c|c|c|c|c|c|c|}
\hline Threshold of CAC score evaluated & No of studies & No of participants evaluated & & $\mathrm{RR}(95 \% \mathrm{CI})$ & $I^{2}$ & $t^{2}$ \\
\hline \multicolumn{7}{|l|}{ CAC score $=0$ as reference group: } \\
\hline \multirow[t]{2}{*}{ Score $>0$ vs 0} & $87(12-21,23-30)$ & 30,057 & Random effects & $5.71[3.98 ; 8.19]$ & $65 \%$ & 0.2778 \\
\hline & & & Fixed effects & $6.58[5.58 ; 7.76]$ & & \\
\hline \multirow[t]{2}{*}{ Score $>/ 100$ vs 0} & $7(13,18-21,24,28)$ & 9434 & Random effects & $9.57[6.87 ; 13.33]$ & $23 \%$ & 0.0433 \\
\hline & & & Fixed effects & $9.71[7.70 ; 12.25]$ & & \\
\hline \multirow[t]{2}{*}{ Score $>/ 400$ vs 0} & $9(13,18-21,23-25,27)$ & 8577 & Random effects & $8.30[4.95 ; 13.90]$ & $77 \%$ & 0.4083 \\
\hline & & & Fixed effects & $9.21[7.47 ; 11.36]$ & & \\
\hline \multicolumn{7}{|l|}{ CAC score $<100$ as reference group: } \\
\hline \multirow[t]{2}{*}{ Score $>/ 100$ vs $<100$} & $7(13,18-21,24,28)$ & 13,198 & Random effects & $4.09[2.85 ; 5.89]$ & $79 \%$ & 0.1652 \\
\hline & & & Fixed effects & $4.81[4.19 ; 5.53]$ & & \\
\hline \multirow[t]{2}{*}{ Score $>/ 400$ vs $<100$} & $6(13,18-21,24)$ & 10,762 & Random effects & $5.08[3.52 ; 7.34]$ & $75 \%$ & 0.1389 \\
\hline & & & Fixed effects & $6.03[5.16 ; 7.05]$ & & \\
\hline \multicolumn{7}{|l|}{ CAC score $<400$ as reference group: } \\
\hline \multirow[t]{2}{*}{ Score $>/ 400$ vs $<400$} & $9(13,18-21,23-25,27)$ & 15,368 & Random effects & $3.30[2.41 ; 4.51]$ & $83 \%$ & 0.1746 \\
\hline & & & Fixed effects & $3.83[3.41 ; 4.31]$ & & \\
\hline
\end{tabular}


A

Study

1 Al-Mallah, 2014

2 Becker, 2005

3 Bom, 2016

4 Chaikriangkrai, 2015

5 Dedic, 2011

6 Detrano, 1996

7 Engbers, 2016

8 Hadamitzky, 2011

9 Hou, 2012

10 Hulten, 2014

11 Keelan, 2001

12 Kunita, 2014

13 Lee, 2016

14 Liu, 2013

15 Naya, 2013

16 Parma, 2016

17 Schenker, 2008

18 Schmermund, 2004
CAC $>0 \quad$ CAC $=0$

Events Total Events Total

$\begin{array}{rc}119 & 3767 \\ 78 & 736 \\ 21 & 812 \\ 25 & 451 \\ 28 & 266 \\ 20 & 393 \\ 257 & 3582 \\ 45 & 1400 \\ 307 & 1640 \\ 31 & 662 \\ 21 & 256 \\ 34 & 462 \\ 65 & 739 \\ 54 & 85 \\ 42 & 499 \\ 108 & 349 \\ 44 & 408 \\ 39 & 193 \\ & 16700 \\ 0.2749, & <<0.01\end{array}$

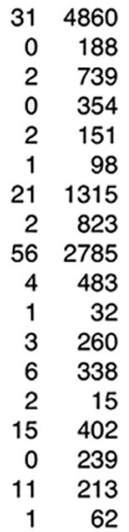

13357

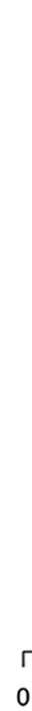

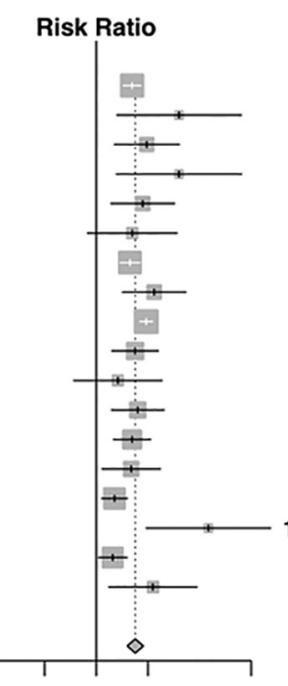

RR

95\%-Cl Weight

$4.95 \quad[3.34 ; 7.34] \quad 10.8 \%$

$40.18[2.50 ; 645.13] \quad 1.5 \%$

$9.56 \quad[2.25 ; 40.62] \quad 4.2 \%$

40.04 [2.45; 655.47] $1.5 \%$

7.95 [1.92; 32.90] $4.3 \%$

$4.99 \quad[0.68 ; 36.71] \quad 2.6 \%$

$4.49 \quad[2.89 ; 6.98] \quad 10.5 \%$

$13.23 \quad[3.22 ; 54.38] \quad 4.3 \%$

$9.31 \quad[7.05 ; 12.30] \quad 11.5 \%$

$5.65 \quad[2.01 ; 15.91] \quad 6.1 \%$

$2.62[0.37 ; 18.86] \quad 2.6 \%$

$6.38 \quad[1.98 ; 20.56] \quad 5.4 \%$

$4.95 \quad[2.17 ; 11.32] \quad 7.5 \%$

$4.76 \quad[1.30 ; 17.49] \quad 4.8 \%$

$2.26 \quad[1.27 ; 4.01] \quad 9.4 \%$

$48.70[9.29 ; 2380.97] \quad 1.5 \%$

$2.09 \quad[1.10 ; 3.96] \quad 8.9 \%$

$12.53 \quad[1.76 ; 89.32] \quad 2.7 \%$

$5.71[3.98 ; 8.19] \quad 100.0 \%$
B

Study
$C A C>0 \quad C A C=0$

Events Total Events Total

$\begin{array}{ll}1 & \text { Al-Mallah, 2014 } \\ 2 & \text { Becker, 2005 } \\ 3 & \text { Bom, 2016 } \\ 4 & \text { Chaikriangkrai, } 2015 \\ 5 & \text { Dedic, 2011 } \\ 6 & \text { Detrano, 1996 } \\ 7 & \text { Engbers, 2016 } \\ 8 & \text { Hadamitzky, 2011 } \\ 9 & \text { Hulten, 2014 } \\ 10 & \text { Keelan, 2001 } \\ 11 & \text { Naya, 2013 } \\ 12 & \text { Parma, 2016 } \\ 13 & \text { Schenker, 2008 } \\ 14 & \text { Schmermund, } 2004\end{array}$

Random effects model

Heterogeneity: $l^{2}=16 \%, \tau^{2}=0.0483, p=0.28$
13774

$\begin{array}{rrrr}119 & 3767 & 31 & 4860 \\ 78 & 736 & 0 & 188 \\ 7 & 812 & 0 & 739 \\ 16 & 451 & 0 & 354 \\ 10 & 266 & 0 & 151 \\ 20 & 393 & 1 & 98 \\ 152 & 3582 & 19 & 1315 \\ 10 & 1400 & 2 & 823 \\ 14 & 662 & 3 & 483 \\ 21 & 256 & 1 & 32 \\ 27 & 499 & 8 & 402 \\ 14 & 349 & 0 & 239 \\ 44 & 408 & 11 & 213 \\ 5 & 193 & 0 & 62\end{array}$

9959

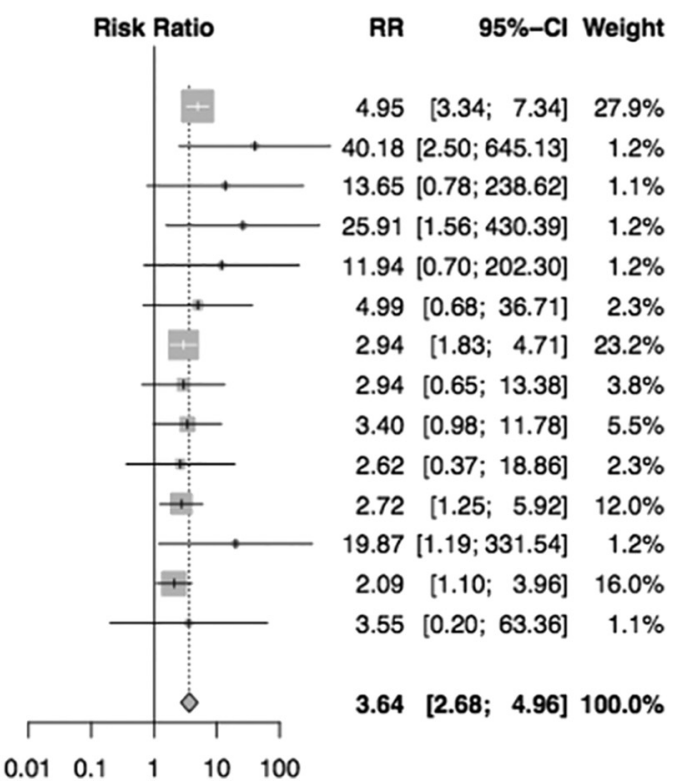

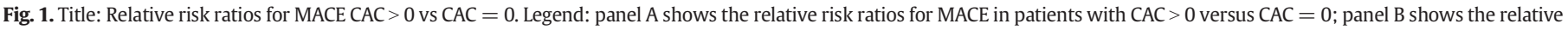

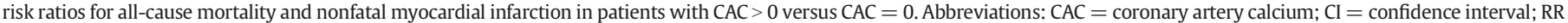
$=$ relative risk

consists of coronary revascularizations, we analyzed the value of CAC scanning on 'hard endpoints', i.e. the composite of all-cause mortality and nonfatal myocardial infarction.

Pooled results from 5 population-based cohort studies with 6739 low-risk women showed that CAC was associated with increased risk of atherosclerotic CVD [7]. In that study, patients with positive CAC scores had a hazard ratio of 2.04 as compared with patients without any CAC. Another study found no significant differences between men and women for mild- to high-risk CAC scores, suggesting an equivalent ability to stratify CVD risk by gender [32]. The data in the studies we included did not allow us to stratify by gender. In a recent meta-analysis of stable patients presenting with acute symptoms, absence of CAC was associated with significantly lower risk of future cardiovascular events [33]. Although these meta-analyses included different study populations, their findings are consistent with our data on symptomatic patients with stable symptoms.
In our analyses we used a CAC score threshold of $>400$, which is similar to the thresholds used in the studies we included. However, the recently updated SCCT expert consensus statement for CAC scoring recommends using a threshold of CAC score $>300$ [34].

The persistent interest in the predictive utility of CAC testing is not surprising. In the asymptomatic population, CAC scoring has proven to be a reliable method for estimating calcified plaque burden and identifying future cardiovascular events. When added to existing risk prediction algorithms, CAC testing was shown to reliably predict future CVD events [35]. CAC testing was found to be cost-effective in the assessment of intermediate risk patients [36].

CAC testing can preclude the need for further downstream testing [37,38], and both screening and identification of CAC abnormalities may lead to improvements in cardiovascular risk factors [39]. In addition, there is evidence indicating that CAC testing has positive impact on adherence of statin therapy and lifestyle changes [40,41]. Given its 
low radiation dose and relatively low-cost (usually less than $\$ 100$ ), it is plausible that CAC scoring will play an increasingly important role in health care management for the asymptomatic population $[42,43]$.

The diagnostic value of CAC scanning in symptomatic patients is increasingly being investigated. Compared to CCTA, CAC testing has the additional benefit of being rapid and non-invasive, not requiring venipuncture, and being easily accessible. The prognostic value of CAC compared to functional stress testing among stable patients with suspected $\mathrm{CAD}$ was recently investigated in a prospective trial. In that trial, most patients who experienced clinical events presented with positive CAC scans, while only a minority exhibited functional stress test abnormalities. Still, as a result of the higher specificity of the abnormal functional test, both tests were found to have similar discriminatory abilities [44].

As stated previously, it is plausible that CAC scoring will play an increasingly important role in health care management for the asymptomatic population $[42,43]$. Similarly, the diagnostic role of CAC testing in symptomatic patients might gain greater clinical utility. Although CCTA may be done in many symptomatic patients, preangiographic CAC scoring may be helpful in the decision to proceed to angiography or to defer the procedure. But in particular in the primary care setting, CAC testing may prove to be very useful, since referral may not be indicated in men and women with mild symptoms and relatively low CAC scores.

As stated previously, current literature provides solid evidence that absence of CAC is associated with favorable prognosis in asymptomatic individuals [45,2]. The same evidence is now emerging that this holds true in symptomatic patients as well [31,46-49]. Nonetheless, the results of different studies addressing outpatients with a CAC score of zero appear equivocal [47,50]. For instance, Gottlieb et al. () found that absence of CAC did not exclude the presence of obstructive stenoses or the need for revascularization in their population with suspected CAD and high pretest probability. Separate analyses from a symptomatic cohort trial showed that CAC absence did significantly reduce, but did not fully eliminate the occurrence of obstructive CAD [47]. Still, the absence of CAC significantly lowered the risk of adverse future events in all reports on CAC scanning. Of course, no normal test outcome - not even normal coronary angiography - can guarantee a completely event-free in all patients and doctors must remain vigilant when CAD is still suspected on clinical grounds.

In total, 13 of the CAC studies that we analyzed, reported on subsequent coronary revascularizations, either percutaneous coronary intervention or coronary artery bypass graft. Of these studies, 11 included only late, from 60 days to 6 months post coronary CCTA, revascularizations. Exclusion of early revascularizations from survival analysis could minimize verification bias [51]. Of course, patients with significant coronary lumen stenosis $(\geq 50 \%)$ by CCTA are more likely to undergo invasive angiography and revascularization early post-CCTA in clinical practice. Late revascularizations, on the other hand, are less frequently associated with CCTA findings and more often triggered by the symptomatology of patients. Three studies that we reported did not separately consider early and late coronary revascularizations. When we studied the relation between CAC scores and all-cause mortality and myocardial infarction, after the exclusion of events as revascularizations and hospitalizations, we found that patients with positive CAC scores had a 5-fold increased risk. Appropriate preventive treatment of patients with coronary calcium should reduce their risk of future complications.

\subsection{Strengths \&'limitations}

Our meta-analysis is not without limitations. We recognize the importance of the specific usefulness of CAC scoring in patients with low to intermediate CAD probability and with non-specific or atypical chest pain. Unfortunately, since the majority of studies did not specify type of chest pain, it was not possible to analyze this separately and thus better estimate the usefulness of CAC score in symptomatic patients. Second, assessment of the large number of studies that were included in our analyses showed some heterogeneity among the populations under study. This was accounted for by applying random effects models. Although the findings of most studies that we included were concordant, our findings might not be generalizable to populations that were not represented in this meta-analysis. Third, in the analysis of the relation between CAC scores and hard events we were not able to adjust for several important variables, such as optimal medical treatment and achieved LDL-cholesterol and glucose goals. Hence, our calculated increased risk of all-cause mortality and myocardial infarction might be an overestimation. This is, on the other hand and to the best of our knowledge, the largest meta-analysis to report the on prognostic value of CAC in stable symptomatic patients. Our data and results confirm and strengthen the strong association between CAC and prognosis in the symptomatic patient population. However, the strong associations between CAC and increased risk as reported here may be modified by proper preventive measures in patients found to be at high risk on the basis of their calcium score.

\section{Conclusion}

On the basis of our analyses on over 34,000 stable, symptomatic patients with suspected CAD, we conclude that increased levels of coronary artery calcium are strongly and independently associated with increased risk for major adverse cardiac events. In these patients, the risk for cardiac events increased with greater CAC scores. The findings are clinically relevant for the large group of symptomatic patients and, although a multicenter randomized trial will be needed to assess the exact utility and incremental predictive value of calcium testing, our analyses indicate that CAC scanning should be helpful in clinical decision making in a considerable number of stable patients with chest pain.

Supplementary data to this article can be found online at https://doi. org/10.1016/j.ijcard.2019.06.003.

\section{Funding}

This research did not receive any specific grant from funding agencies in the public, commercial, or not-for-profit sectors.

\section{Declaration of Competing Interest}

M.S.L., R.v.D., M.H., J.A.C.L., S.E.H., J.W.D.: nothing to disclose; D.R-H.: supported by an unrestricted grant from the Bronovo research fund.

\section{Acknowledgements}

The authors wish to thank Wichor Bramer, Medical librarian Erasmus University Rotterdam, for his support with the literature search.

\section{References}

[1] A.S. Agatston, W.R. Janowitz, F.J. Hildner, N.R. Zusmer, M. Viamonte Jr., R. Detrano Quantification of coronary artery calcium using ultrafast computed tomography, J. Am. Coll. Cardiol. 15 (1990) 827-832.

[2] A.D. Gepner, R. Young, J.A. Delaney, et al., Comparison of coronary artery calcium presence, carotid plaque presence, and carotid intima-media thickness for cardiovascular disease prediction in the multi-ethnic study of atherosclerosis, Circ. Cardiovasc. Imaging 8 (2014).

[3] R.L. McClelland, N.W. Jorgensen, M. Budoff, et al., 10-Year coronary heart disease risk prediction using coronary artery calcium and traditional risk factors: derivation in the MESA (multi-ethnic study of atherosclerosis) with validation in the HNR (Heinz Nixdorf recall) Study and the DHS (Dallas Heart Study), J. Am. Coll. Cardiol. 66 (2015) 1643-1653.

[4] D.C. Goff Jr., D.M. Lloyd-Jones, G. Bennett, et al., 2013 ACC/AHA guideline on the assessment of cardiovascular risk: a report of the American College of Cardiology/ 
American Heart Association Task Force on Practice Guidelines, Circulation 129 (2014) S49-S73.

[5] M.J. Budoff, S. Achenbach, R.S. Blumenthal, et al., Assessment of coronary artery disease by cardiac computed tomography: a scientific statement from the American Heart Association Committee on Cardiovascular Imaging and Intervention, Council on Cardiovascular Radiology and Intervention, and Committee on Cardiac Imaging, Council on Clinical Cardiology, Circulation 114 (2006) 1761-1791.

[6] K. Chaikriangkrai, H.Y. Jhun, G. Palamaner Subash Shantha, et al., Coronary artery calcium score as a predictor for incident stroke: systematic review and metaanalysis, Int. J. Cardiol. 236 (2017) 473-477.

[7] M. Kavousi, C.S. Desai, C. Ayers, et al., Prevalence and prognostic implications of coronary artery calcification in low-risk women: a meta-analysis, JAMA 316 (2016) 2126-2134.

[8] C.K. Kramer, B. Zinman, J.L. Gross, et al., Coronary artery calcium score prediction of all cause mortality and cardiovascular events in people with type 2 diabetes: systematic review and meta-analysis, BMJ (Online) 346 (2013).

[9] M.J. Pletcher, J.A. Tice, M. Pignone, W.S. Browner, Using the coronary artery calcium score to predict coronary heart disease events: a systematic review and metaanalysis, Arch. Intern. Med. 164 (2004) 1285-1292.

[10] D.F. Stroup, J.A. Berlin, S.C. Morton, et al., Meta-analysis of observational studies in epidemiology: a proposal for reporting. Meta-analysis Of Observational Studies in Epidemiology (MOOSE) group, JAMA 283 (2000) 2008-2012.

[11] J.A. Hayden, P. Cote, C. Bombardier, Evaluation of the quality of prognosis studies in systematic reviews, Ann. Intern. Med. 144 (2006) 427-437.

[12] E. Hulten, M.S. Bittencourt, B. Ghoshhajra, et al., Incremental prognostic value of coronary artery calcium score versus CT angiography among symptomatic patients without known coronary artery disease, Atherosclerosis 233 (2014) 190-195.

[13] M. Hadamitzky, R. Distler, T. Meyer, et al., Prognostic value of coronary computed tomographic angiography in comparison with calcium scoring and clinical risk scores, Circ. Cardiovasc. Imaging 4 (2011) 16-23.

[14] M.H. Al-Mallah, W. Qureshi, F.Y. Lin, et al., Does coronary CT angiography improve risk stratification over coronary calcium scoring in symptomatic patients with suspected coronary artery disease? Results from the prospective multicenter international CONFIRM registry, Eur. Heart J. Cardiovasc. Imaging 15 (2014) 267-274.

[15] A. Becker, A. Knez, C. Becker, et al., Prediction of serious cardiovascular events by determining coronary artery calcification measured by multi-slice computed tomography, Dtsch. Med. Wochenschr. 130 (2005) 2433-2438.

[16] M.J. Bom, P.M. Van der Zee, F.M. Van der Zant, R.J.J. Knol, J.H. Cornel, Independent prognostic value of coronary artery calcium score and coronary computed tomography angiography in an outpatient cohort of low to intermediate risk chest pain patients, Neth. Hear. J. 24 (2016) 332-342.

[17] K. Chaikriangkrai, P. Velankar, R. Schutt, et al., Additive prognostic value of coronary artery calcium score over coronary computed tomographic angiography stenosis assessment in symptomatic patients without known coronary artery disease, Am. J. Cardiol. 115 (2015) 738-744.

[18] A. Dedic, T.S.S. Genders, B.S. Ferket, et al., Stable angina pectoris: head-to-head comparison of prognostic value of cardiac CT and exercise testing, Radiology 261 (2011) 428-436.

[19] E.M. Engbers, J.R. Timmer, J.P. Ottervanger, M. Mouden, S. Knollema, P.L. Jager, Prognostic value of coronary artery calcium scoring in addition to single-photon emission computed tomographic myocardial perfusion imaging in symptomatic patients, Circ. Cardiovasc. Imaging 9 (2016).

[20] Z.H. Hou, B. Lu, Y. Gao, et al., Prognostic value of coronary CT angiography and calcium score for major adverse cardiac events in outpatients, JACC Cardiovasc. Imaging 5 (2012) 990-999.

[21] E. Kunita, H. Yamamoto, T. Kitagawa, et al., Prognostic value of coronary artery calcium and epicardial adipose tissue assessed by non-contrast cardiac computed tomography, Atherosclerosis 233 (2014) 447-753.

[22] S.W. Kwon, Y.J. Kim, J. Shim, et al., Coronary artery calcium scoring does not add prognostic value to standard 64-section CT angiography protocol in lowrisk patients suspected of having coronary artery disease, Radiology 259 (2011) 92-99.

[23] H. Lee, Y.E. Yoon, J.B. Park, et al., The incremental prognostic value of cardiac computed tomography in comparison with single-photon emission computed tomography in patients with suspected coronary artery disease, PLoS One 11 (2016).

[24] Y.C. Liu, Z. Sun, P.K. Tsay, et al., Significance of coronary calcification for prediction of coronary artery disease and cardiac events based on 64-slice coronary computed tomography angiography, Biomed. Res. Int. 2013 (2013).

[25] M. Naya, V.L. Murthy, C.R. Foster, et al., Prognostic interplay of coronary artery calcification and underlying vascular dysfunction in patients with suspected coronary artery disease, J. Am. Coll. Cardiol. 61 (2013) 2098-2106.

[26] Z. Parma, R. Parma, J. Brzoska, Prognostic Value of Coronary Artery Calcium Score in Patients with Symptoms Suggestive of Coronary Artery Disease. Results from the Silesian Calcium Score (SILICAS) .... Polskie Archiwum ..., 2016

[27] M.P. Schenker, S. Dorbala, E.C.T. Hong, et al., Interrelation of coronary calcification, myocardial ischemia, and outcomes in patients with intermediate likelihood of coronary artery disease: a combined positron emission tomography/computed tomography study, Circulation 117 (2008) 1693-1700.

[28] A. Schmermund, A. Stang, S. Mohlenkamp, et al., Prognostic value of electron-beam computed tomography-derived coronary calcium scores compared with clinical parameters in patients evaluated for coronary artery disease - prognostic value of EBCT in symptomatic patients, Zeitschrift Fur Kardiologie 93 (2004) 696-705.
[29] R. Detrano, T. Hsiai, S. Wang, et al., Prognostic value of coronary calcification and angiographic stenoses in patients undergoing coronary angiography, J. Am. Coll. Cardiol. 27 (1996) 285-290.

[30] P.C. Keelan, L.F. Bielak, K. Ashai, et al., Long-term prognostic value of coronary calcification detected by electron-beam computed tomography in patients undergoing coronary angiography, Circulation 104 (2001) 412-417.

[31] A. Sarwar, L.J. Shaw, M.D. Shapiro, et al., Diagnostic and prognostic value of absence of coronary artery calcification, JACC Cardiovasc. Imaging 2 (2009) 675-688.

[32] A. Bellasi, C. Lacey, A.J. Taylor, et al., Comparison of prognostic usefulness of coronary artery calcium in men versus women (results from a meta- and pooled analysis estimating all-cause mortality and coronary heart disease death or myocardial infarction), Am. J. Cardiol. 100 (2007) 409-414.

[33] K. Chaikriangkrai, G. Palamaner Subash Shantha, H.Y. Jhun, et al., Prognostic value of coronary artery calcium score in acute chest pain patients without known coronary artery disease: systematic review and meta-analysis, Ann. Emerg. Med. 68 (2016) 659-670.

[34] H. Hecht, M.J. Blaha, D.S. Berman, et al., Clinical indications for coronary artery calcium scoring in asymptomatic patients: expert consensus statement from the Society of Cardiovascular Computed Tomography, J. Cardiovasc. Comput. Tomogr. 11 (2017) 157-168

[35] T.S. Polonsky, R.L. McClelland, N.W. Jorgensen, et al., Coronary artery calcium score and risk classification for coronary heart disease prediction, JAMA 303 (2010) 1610-1616.

[36] K. Nasir, M.S. Bittencourt, M.J. Blaha, et al., Implications of coronary artery calcium testing among statin candidates according to American College of Cardiology/American Heart Association Cholesterol Management Guidelines: MESA (multi-ethnic study of atherosclerosis), J. Am. Coll. Cardiol. 66 (2015) 1657-1668.

[37] R.S. Blumenthal, C.W. Hwang, K. Nasir, Selective use of coronary artery calcium screening: worth the cost? J. Am. Coll. Cardiol. 54 (2009) 1268-1270.

[38] L.J. Shaw, J.K. Min, M. Budoff, et al., Induced cardiovascular procedural costs and resource consumption patterns after coronary artery calcium screening: results from the EISNER (early identification of subclinical atherosclerosis by noninvasive imaging research) study, J. Am. Coll. Cardiol. 54 (2009) 1258-1267.

[39] A. Rozanski, H. Gransar, LJ. Shaw, et al. Impact of coronary artery calcium scanning on coronary risk factors and downstream testing: the EISNER (early identification of subclinical atherosclerosis by noninvasive imaging research) prospective randomized trial, J. Am. Coll. Cardiol. 57 (2011) 1622-1632.

[40] N.K. Kalia, L.G. Miller, K. Nasir, R.S. Blumenthal, N. Agrawal, M.J. Budoff, Visualizing coronary calcium is associated with improvements in adherence to statin therapy, Atherosclerosis 185 (2006) 394-399.

[41] A.J. Taylor, J. Bindeman, I. Feuerstein, et al., Community-based provision of statin and aspirin after the detection of coronary artery calcium within a community-based screening cohort, J. Am. Coll. Cardiol. 51 (2008) 1337-1341.

[42] M.J. Budoff, L.J. Shaw, S.T. Liu, et al., Long-term prognosis associated with coronary calcification: observations from a registry of 25,253 patients, J. Am. Coll. Cardiol. 49 (2007) 1860-1870

[43] K. Alluri, P.H. Joshi, T.S. Henry, R.S. Blumenthal, K. Nasir, M.J. Blaha, Scoring of coronary artery calcium scans: history, assumptions, current limitations, and future directions, Atherosclerosis 239 (2015) 109-117.

[44] M.J. Budoff, T. Mayrhofer, M. Ferencik, et al., The prognostic value of coronary artery calcium in the PROMISE study, Circulation 136 (21) (2017) 1993-2005.

[45] J. Yeboah, R.L. McClelland, T.S. Polonsky, et al., Comparison of novel risk markers for improvement in cardiovascular risk assessment in intermediate-risk individuals, JAMA 308 (2012) 788-795.

[46] D. Rijlaarsdam-Hermsen, D. Kuijpers, P.R.M. van Dijkman, Diagnostic and prognostic value of absence of coronary artery calcification in patients with stable chest symptoms, Neth. Hear. J. 19 (2011) 223-228.

[47] T.C. Villines, E.A. Hulten, L.J. Shaw, et al., Prevalence and severity of coronary artery disease and adverse events among symptomatic patients with coronary artery calcification scores of zero undergoing coronary computed tomography angiography: results from the CONFIRM (coronary CT angiography evaluation for clinical outcomes: an international multicenter) registry, J. Am. Coll. Cardiol. 58 (2011) 2533-2540.

[48] M. Mouden, J.R. Timmer, S. Reiffers, et al., Coronary artery calcium scoring to exclude flow-limiting coronary artery disease in symptomatic stable patients at low or intermediate risk, Radiology 269 (2013) 77-83.

[49] F. Nabi, S.M. Chang, C.M. Pratt, et al., Coronary artery calcium scoring in the emergency department: identifying which patients with chest pain can be safely discharged home, Ann. Emerg. Med. 56 (2010) 220-229.

[50] Y.J. Kim, J. Hur, H.J. Lee, et al., Meaning of zero coronary calcium score in symptomatic patients referred for coronary computed tomographic angiography, Eur. Heart J. Cardiovasc. Imaging 13 (2012) 776-785.

[51] M. Hadamitzky, B. Freissmuth, T. Meyer, et al., Prognostic value of coronary computed tomographic angiography for prediction of cardiac events in patients with suspected coronary artery disease, JACC Cardiovasc. Imaging 2 (2009) 404-411. 